\title{
DISEÑO DE UN SISTEMA DE SEMAFORIZACIÓN ELECTRÓNICO
}

\author{
David A. Luz-Luz ${ }^{1}$, John E. Mendigaña-Figueredo ${ }^{1}$
}

${ }^{1}$ Tecnólogo, Facultad Tecnológica, Universidad Distrital Francisco José de Caldas, Colombia

Correo electrónico: stewartdetroya@hotmail.com

Recibido: 15 de abril del 2013. Aprobado: 10 de junio del 2013.

Cómo citar este artículo: D. A. Luz-Luz y J. E. Mendigaña-Figueredo, "Diseño de un sistema de semaforización electrónico”. Ingeniería Solidaria, Vol. 9, №. 16, pp. 57-64, Dic., 2013.

Resumen. El artículo es producto del proyecto de grado titulado "Diseño de un sistema de semaforización electrónico desarrollado en la empresa de gaseosas Colombiana del Sur” y se sustentó en el 2010 en la Facultad Tecnológica de la Universidad Distrital Francisco José de Caldas. El sistema está conformado por dos sensores TrafiCam ${ }^{\circledR}$ (sensores de presencia vehicular), basados en la tecnología de vídeo detección probada a nivel mundial, que cuenta con una cámara (blanco y negro) cmos e interfaz de comunicación por RS485 o UsB, junto con un sistema de control programado y diseñado con microcontroladores PSoC (Programable System on Chip) que se adapta a las necesidades de las TrafiCam y los requerimientos para el flujo adecuado de vehículos y peatones dentro de la compañía. El aporte más importante producto de esta investigación es que las cámaras que hacen parte del sistema diseñado tienen la capacidad de detección en tiempo real de objetos (vehículos) en reposo y movimiento que intervengan en el bucle virtual.

Palabras clave: PSoC, RS485, semaforización, TrafiCam ${ }^{\circ}$

\section{Designing an Electronic Traffic Light System}

Abstract. This article is the product of the degree project titled "Design of an electronic traffic light system developed in the Gaseosas Colombianas del Sur company" and was presented and defended in 2010 in the Technology Faculty of the Universidad Distrital Francisco José de Caldas. The system consists of two TrafiCam sensors (vehicle presence sensors), based on video detection technology tested worldwide. It uses a CMOs camera (black and white) and an RS485 or USB communication interface, together with a control system programmed and designed with PSoC (Programmable System on Chip) microcontrollers that adjusts to the needs of the TrafiCams and the requirements for adequate vehicle and pedestrian flow within the company grounds. The most important finding of this research study is that the cameras in the system designed can detect objects (vehicles) that enter the virtual loop, at rest and in movement, in real time.

Keywords: PSoC, RS485, traffic lights, TrafiCam

\section{DESENHO DE UM SISTEMA DE SEMAFORIZAÇÃo ELETRÔNICA}

Resumo. Este artigo é produto do projeto de graduação intitulado "Desenho de um sistema de semaforização eletrônica desenvolvido na empresa de gasosas Colombiana do Sul" e foi defendido em 2010 na Faculdade Tecnológica da Universidade Distrital Francisco José de Caldas. O sistema está conformado por dois sensores TrafiCam ${ }^{\circledR}$ (sensores de presença veicular), baseados na tecnologia de vídeo deteç̧ão provada mundialmente, que conta com uma câmera (preto e branco) cmos e interface de comunicação por RS485 ou pen drive, junto com um sistema de controle programado e desenhado com microcontroladores PSoC (Programable System on Chip) que se adapta às necessidades das TrafiCam e dos requerimentos para o fluxo adequado de veículos e pedestres dentro da companhia. A contribuição mais importante produto desta pesquisa se deve às câmeras que fazem parte do sistema desenhado que têm a capacidade de detecção em tempo real de objetos (veículos) em repouso e movimento que intervenham no lacete virtual.

Palavras-chave: PSoC, RS485, semaforização, TrafiCam . 


\section{Introducción}

Las empresas de gaseosas colombianas poseen problemas para controlar y ordenar el tráfico en la línea de producción, asunto que impacta la productividad y la calidad del producto. Esta tendencia no permite prever la aparición de accidentes laborales, pues debe mejorarse y ordenarse el flujo vehicular. Velázquez [1] indica que los sistemas de control de tráfico, más recientemente los denominados sistemas inteligentes de transporte (ITs, por sus siglas en inglés, Intelligent Transportation Systems), incluyen el sistema de señalización del tráfico y el de semaforización electrónica. El concepto de sistemas de control de tráfico involucra un conjunto de equipamiento computacional de central de tráfico, que por medio de un sistema de telecomunicaciones con los equipos de control local del tráfico (comúnmente denominados controladores) permite monitorear en línea el funcionamiento del tráfico y aplicar distintos planes de regulación.

En el caso que nos ocupa, la semaforización electrónica con detección por TrafiCam ${ }^{\circledR}$ es actualmente una alternativa globalizada fundamental para la reducción de accidentes, mejoramiento del flujo vehicular y detección inteligente de problemas en la vía, permitiendo adaptarse a las condiciones de los sitios de empleo de manera más efectiva.

Entonces los requerimientos de control para las compañías descritas demandan incorporar sistemas versátiles y flexibles. En este sentido, el chip PSoC (Programable System-on-Chip) ofrece una arquitectura flexible a modificaciones en el desarrollo de embedded systems, ${ }^{1}$ ya que permite cambiar las configuraciones de los módulos analógicos o digitales, según nuestras necesidades, o si los resultados obtenidos no son satisfactorios [2-4]. Estas configuraciones se realizan vía software, ofreciendo una ventaja sobre otros microcontroladores de su clase, como por ejemplo la posibilidad de la reconfigurabilidad, entre muchas otras.

Este documento describe la alternativa que, producto de la investigación, se le suma a lo descrito un sistema electrónico para la efectividad del control, y la etapa de potencia que estará acoplada a los semáforos. La estructura del artículo es así: análisis y diseño; adecuación del sistema de tráfico y del sistema electrónico de tráfico; medición y pruebas; resultados y conclusiones.

1 Embedded systems o sistemas embebidos o empotrados, son sistemas computacionales diseñados para realizar una o algunas funciones dedicadas frecuentemente en un sistema de computación en tiempo real.

\section{Antecedentes y diseño}

Velásquez [1], autor del proyecto de grado titulado "Desarrollo de una metodología para el control de la señalización del tráfico y el sistema de semaforización utilizando las líneas de la potencia electrónica PLT (PLC)", explica algunos antecedentes, pero circunscritos a otros contextos de tráfico, concebidos en distintas modalidades de operación o estrategias de control, a saber: actuado, responsivo y adaptativo, como lo define el Departamento de Planeación Nacional de Colombia (DNP):

Control por tiempos fijos. Bajo la operación de un tiempo fijo, el centro de control semafórico envía órdenes con tiempos de ciclo predeterminados. Este control está basado en datos históricos y son apropiados para áreas donde la demanda es predecible; actuado: El controlador opera basado en las demandas de tráfico vehicular o peatonal que registran los detectores, variando los tiempos de verde mínimos y máximos dependiendo del flujo vehicular sin necesidad de estar conectado con la central semafórica; Responsivo: Similar al actuado, pero en este caso el controlador registra los vehículos, los cuenta y le envía esta información a la central semafórica, quien selecciona unos planes de señales predeterminados y le indica al controlador cual utilizar; Adaptativo: Tiene en cuenta los volúmenes instantáneos del tránsito, la densidad y el tiempo de espera consumido en cada sentido de circulación, pero en lugar de seleccionar un plan predeterminado, el sistema lo crea a través de la central semafórica. Los sistemas adaptativos más utilizados a nivel mundial son: SCOOT, SCATS, SITRAFFIC, ITACA, ADIMOT, OPAC.

En tanto, los sistemas de control de tráfico pueden incluir opciones complementarias que optimicen su operación, tales como: Estaciones de Conteos Vehiculares, Estaciones de mediciones de condiciones ambientales, Supervisión mediante cámaras de video (CCTV), Señalización variable (información a conductores y peatones). En general, apoyan la gestión de tráfico, ya que permite a los operadores del sistema disponer de información actualizada de la situación de la red, entregar indicaciones de las condiciones del tránsito a los usuarios y adoptar acciones ante eventos no programables. Como se observa, los ITs incorporan muchas tecnologías para predecir y evitar problemas como la congestión de tránsito, el mejoramiento de la seguridad vial, la información al viajero y protección medio-ambiental.

Este "matrimonio entre los avances en tecnologías de información y sistemas de comunicación con los vehículos y redes de caminos que forman parte del sistema de transporte" definen "la optimización de las funciones propias de los elementos básicos del Tránsito - Infraes- 
tructura Vial (calles y caminos) y Vehículos - mediante la aplicación de tecnologías avanzadas que interrelacionan tales elementos", [1, p. 15]. Esta investigación se concentrará en la capa física compuesta por los sistemas y las tecnologías, en coherencia con la definición de los servicios o aplicaciones de los sistemas inteligentes de transporte (ITS). La figura 1 muestra el diseño de la solución propuesta.

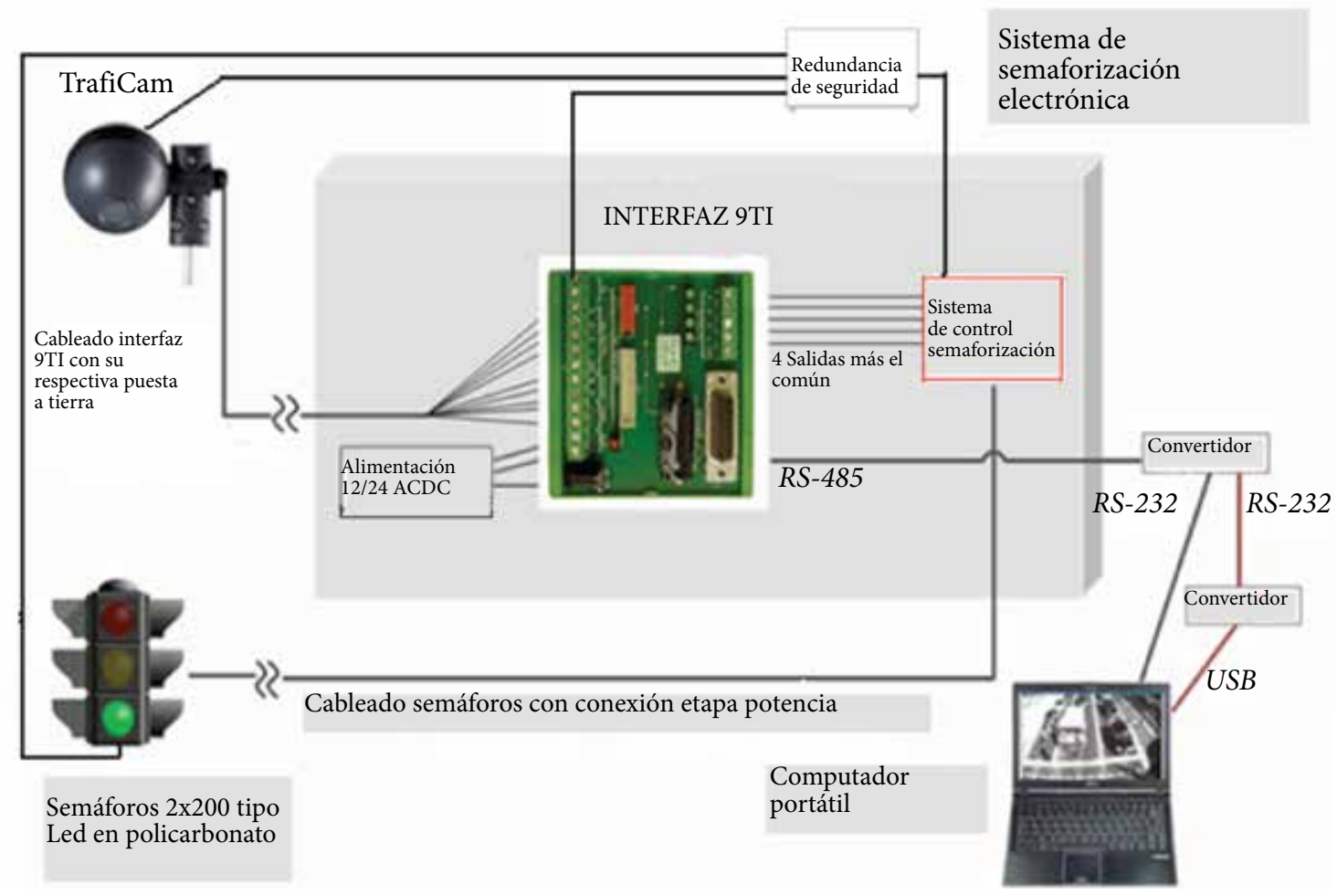

Figura 1. Diseño macro solución

Fuente: los autores

En tanto que la figura 2, indica el diagrama de las etapas a implementar.

\section{Detección vehicular}

\section{Código binario}

\section{Control electrónico de semaforización y sistema} redundante de fallos con visualización en LCD

\section{Etapa de potencia}

\section{Semáforos}

Figura 2. Diagrama del sistema

Fuente: los autores 


\section{Montaje experimental}

\subsection{Adecuación sistema de tráfico}

Se realiza un análisis del flujo vehicular, en el que se encuentra un alto tráfico de montacargas, ingresando por dos puertas a la línea de producción, a las que se piensa instalar unas cortinas plásticas, tal y como se muestra en la figura 3.

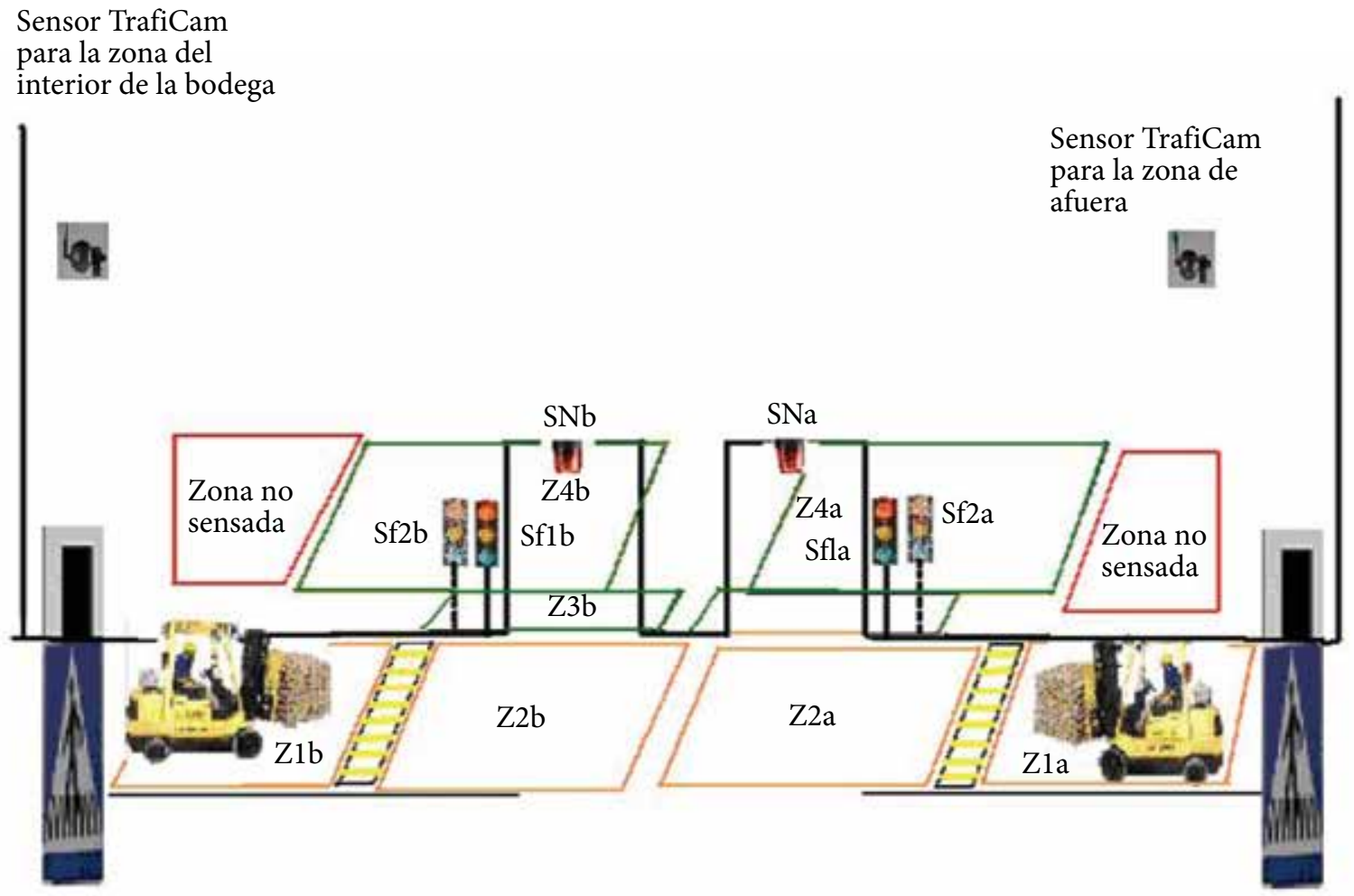

Figura 3. Vista frontal de la línea de producción

Fuente: los autores

Dado que se observa la necesidad de delimitar las zonas para el estacionamiento de vehículos (en rojo en la figura 3), dónde deben detenerse, y las zonas por las que deben transitar los peatones, así como la importancia de abrir una puerta, en la parte izquierda de la figura 3 , a fin de habilitar el tránsito peatonal por este costado, esto se define como crítico para un óptimo funcionamiento del sistema y evitar accidentes. Para futuras instalaciones de las cámaras en los puntos más convenientes, con el fin de abarcar las zonas en bucles virtuales configurables (en la figura 3 se observan los bucles de afuera en naranja y los internos de verde), se deciden los sitios de instalación de semáforos para el avistamiento sencillo y ergonómico para conductores y peatones.

\subsection{Adecuación del sistema electrónico de tráfico}

El sistema electrónico lo constituyen las siguientes partes:

a. Detección: constituido por dos cámaras TrafiCam, las cuales son cámaras y detectores cmos en un sensor compacto. Este pequeño sensor controla la presencia de vehículos que se acercan o que esperan en un cruce, mediante el uso de bucles virtuales con inspección de video como se observa en la figura 4. Además puede detectar vehículos día y noche, se puede configurar hasta en ocho zonas, realizar un conteo de vehículos, detectar un contraflujo vehicular y se adapta a cualquier tipo de superficie. Si no fuera posible la detección exacta, TrafiCam ${ }^{\circledR}$ pasa a un estado de "anular". Cuenta con cuatro salidas ópticamente aisladas, con un consumo de corriente 
absorbida máxima de $30 \mathrm{~mA}$. Otra característica por cumplir corresponde con la norma de compatibilidad electromagnética, teniendo presente el ambiente industrial en que se desempeñarán, regulada por la genérica de inmunidad de compatibilidad-Parte
2: Entorno industrial. 50082-2, al igual que la 55022 para "Equipos de tecnología de información-Características de alteraciones de radio-Límites y métodos de medición".

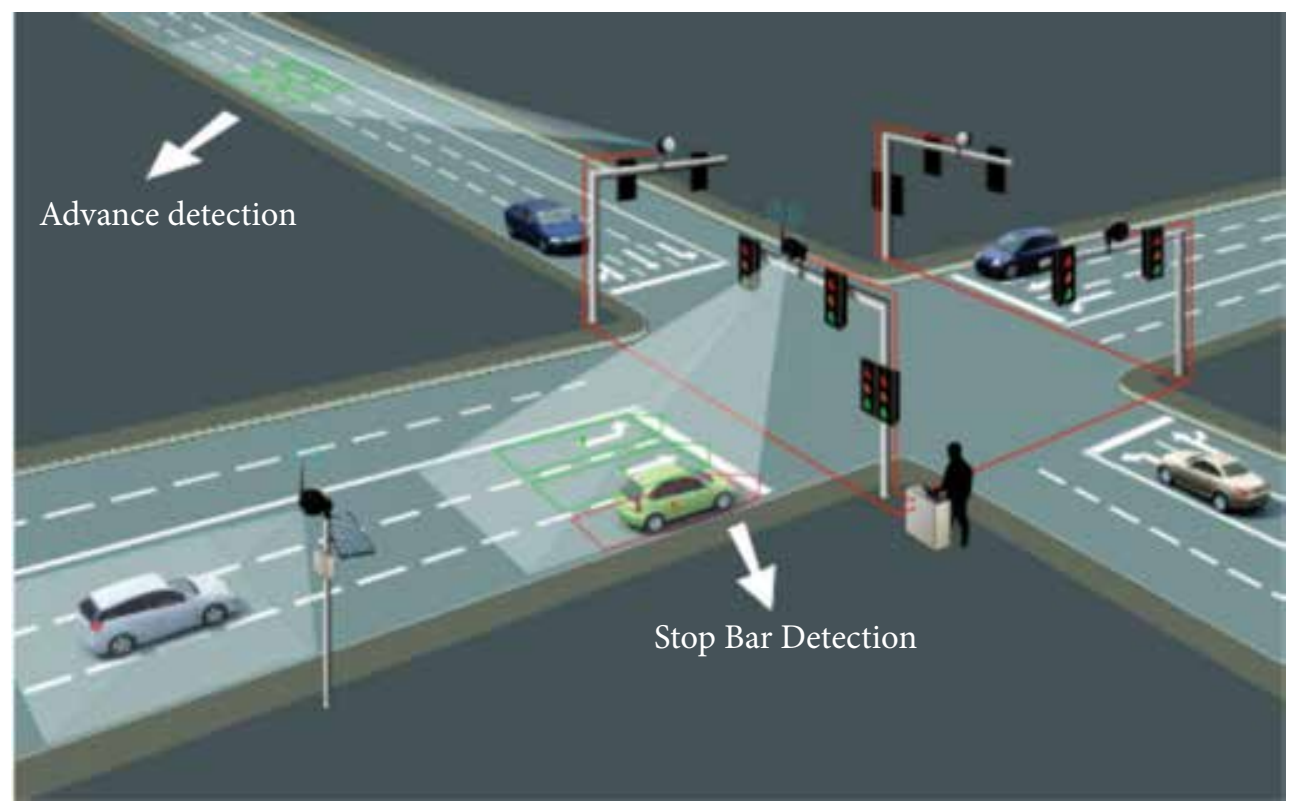

Figura 4. Esquema de cruce vigilado con TrafiCam

Fuente: Traficon [5]

\section{b. Interfaz: cuyas funciones básicas son:}

- Conexión de las salidas de la zona de TrafiCam (las cuales están optoaisladas tipo MOSFET a la controladora, figura 5).

- Proveer de energía a la TrafiCam .

Conexión de un PC portátil vía RS485 o uSB a la TrafiCam para la configuración del sistema y la verificación del mismo.

c. Controlador: diseñado con microcontrolador PSoC CY8C29466 PDIP, encargado de recibir las señales provenientes de la interfaz 9TI y de acuerdo con la combinación de bits que nos proporciona da las órdenes para el manejo del cambio de estados de los semáforos con una lógica previamente estudiada y diseñada para óptimo funcionamiento del sistema teniendo en cuenta que dispone de dos modos de funcionamiento, el modo montacargas donde el control es netamente para los vehículos (montacargas) y personas que transiten por el sitio delimitado y el modo operario que es cuando cambia parte de la lógica básica, ya que no hay un ingreso permitido a la línea de producción por encontrarse esta en mantenimiento o aseo.

Mediante un segundo microcontrolador PSoC que va enlazado al primero se supervisan puntos críticos donde se puedan presentar fallas que afecten el correcto funcionamiento de la semaforización electrónica, como lo serían el manejo de alimentaciones de los semáforos, alimentaciones de las cámaras e interfaces y comprobando el funcionamiento de las tarjetas de potencia mediante la verificación de la respuesta del semáforo, frente al pulso que recibe a la entrada la tarjeta de potencia. Para la información de las fallas o interacción con el usuario se dispone de una LCD $2 \times 16$, en la cual se visualizarán las fallas, ya sean individuales o múltiples, provocando que si el sistema detecta un fallo, este envíe una señal de no operación (todos los semáforos se colocan en rojo) hasta que la falla sea solucionada [4].

En la figura 6 se pueden observar las conexiones de todo el sistema. En los óvalos que se muestran allí se visualiza en mayor escala una explicación más detallada de cómo está compuesta la tarjeta de poten- 


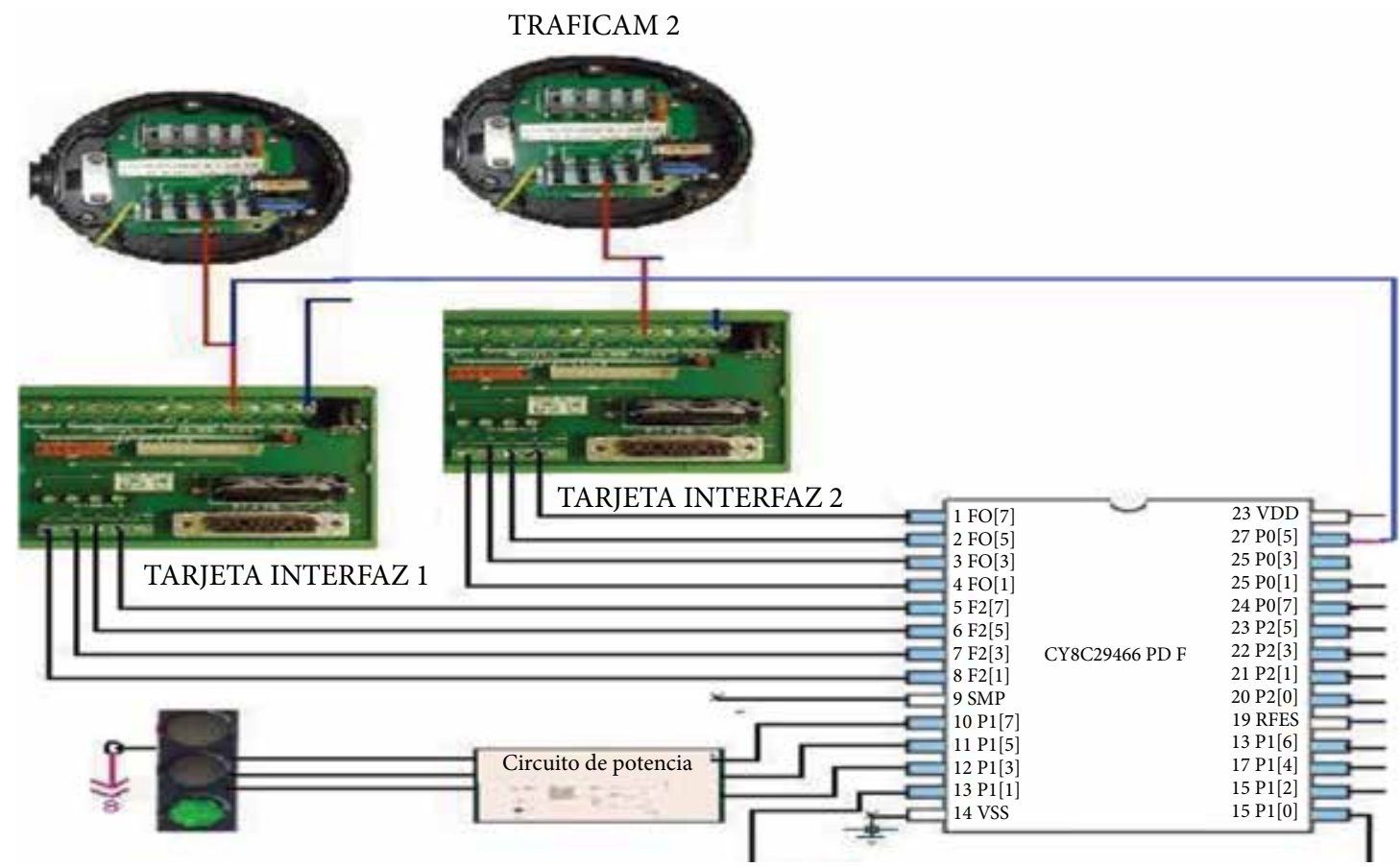

Figura 5. Conexión de las TrafiCam a la tarjeta de interfaz y sistema de control Fuente: los autores

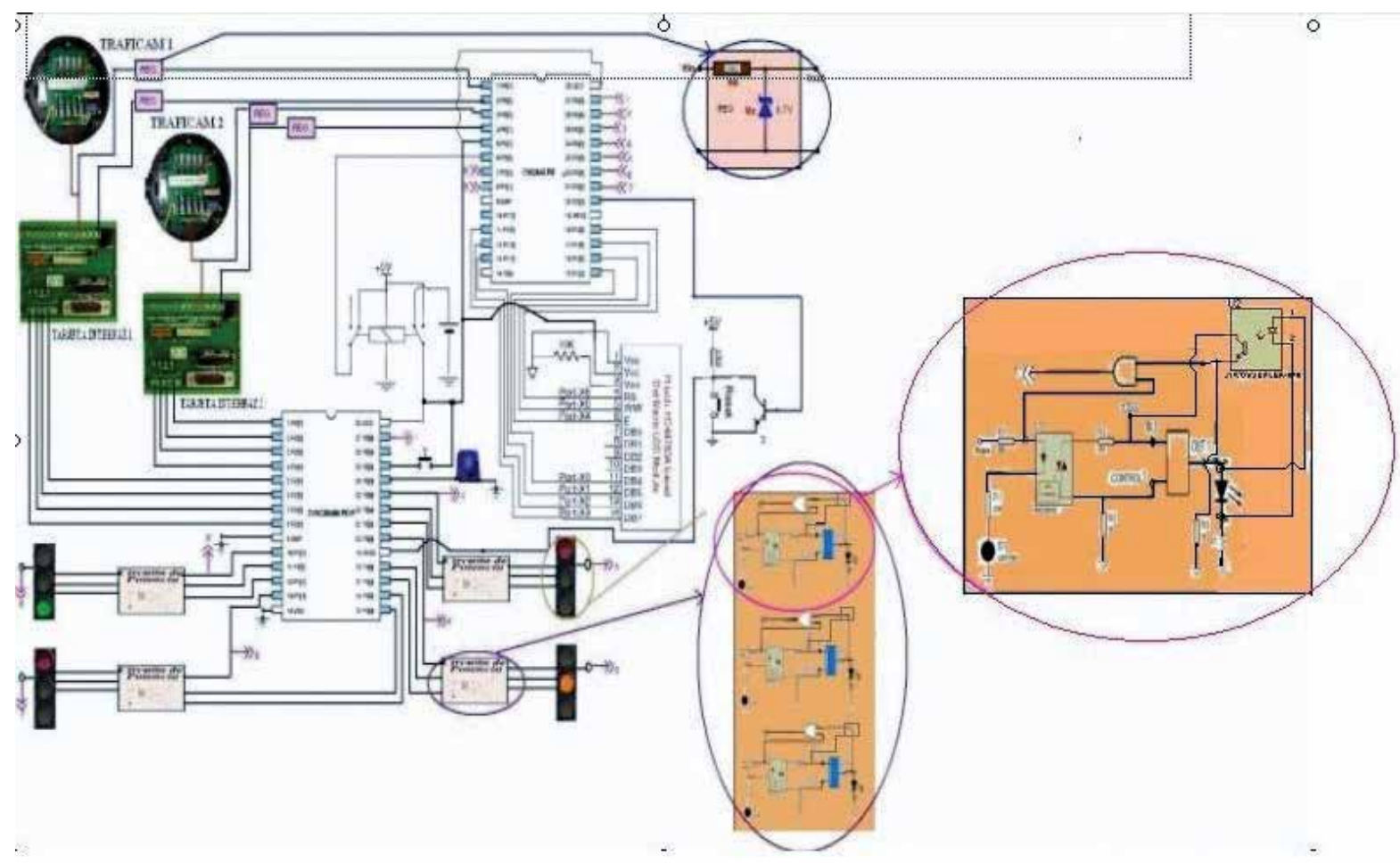

Figura 6. Esquema del diseño completo de semaforización con etapas de control y potencia Fuente: los autores 
cia, así como en la parte superior de la figura se ve el óvalo correspondiente a unos reguladores de voltaje, necesarios para adaptar el voltaje que nos brindan la interfaces y las TrafiCam a un voltaje que pueda ser compatible para las entradas al PSoC. Además, al sistema se le incorpora una batería que proporcionaría voltaje en caso de falla en el suministro principal de alimentación del sistema de control, para así evitar aún más fallas en la operación del conjunto electrónico [5-9].

d. Semáforos: nuevas tecnologías de semaforización que existen en el mercado, como por ejemplo la utilización de leds, son recomendables por contar con ventajas importantes tales como:

- Consumen entre un $60 \%$ a $90 \%$ menos energía, dependiendo del estilo frente al alumbrado convencional incandescente.

- El hecho que se funda un led no implica que el semáforo deje de funcionar.

- Son libres de mantenimiento a corto plazo.

- Opera a una baja temperatura en relación con la luminosidad que proporciona.

- Alta resistencia a la vibración o golpes, debido a que carece de filamento.

- Vida útil extra larga de 50.000 a 100.000 horas.

- Sin radiación UV.

- Alto contraste de luz durante el transcurso del día.

\section{Medición y pruebas}

- Se realizaron, en pruebas iniciales, complementos a la información que se tenía previamente, respecto a los códigos que genera la interfaz cuando se presentan varios eventos de detección en diferentes zonas, pruebas que se hicieron con el vendedor autorizado para Colombia en los laboratorios de la Siemens ${ }^{\circledR}$ [8].

- Se analizó la adaptación del software a las necesidades del flujo de montacargas, ya que fue necesaria una explicación previa de los técnicos de Siemens ${ }^{\varpi}$ acerca del manejo del software, para configurarlo a las necesidades que se tenían; además que este sistema que se diseñó es flexible porque permite a futuro reformas en su funcionamiento [8].

- Se diseñó una maqueta en la que se simuló el espacio y las entradas a la línea de producción, en las cuales se demostró al mantenimiento eléctrico, la manera en que funciona la lógica de los semáforos de acuer- do con las zonas que detectaría la TrafiCam, tanto en el modo automático de montacargas, como en el modo operario (explicados anteriormente en el ítem 2.3 , la parte "c", controlador). Igualmente, se demostró la manera sobre cómo el sistema responde ante fallas y se visualiza mediante LCD, el reporte de una $o$ varias fallas, y continúa trabajando normalmente cuando estas fallas se superan, lo cual satisface la ingeniería de la planta, y muestra la confiabilidad del sistema, pensando en su implementación final.

\section{Resultados}

Se comprende la importancia que tienen las cámaras especiales para tráfico, ya que presentan la posibilidad de adecuar y regular el flujo vehicular, evitando accidentes en vía. También pueden tomar conteos de la cantidad de automotores que cruzan un determinado punto y verificar si hay autos conduciendo en contravía, entre otros. Además, es sobresaliente la manera en que se pueden seleccionar zonas de detección vehicular, aun de vehículos que demoren mucho tiempo en reposo, lo que no sucede con los sensores de presencia en el mercado, donde los únicos más confiables para autos que tengan que estacionarse por un buen tiempo son los sensores de bucle físico, que exigen la realización de obra civil en el piso donde se quieren detectar vehículos para instalar los detectores.

Se prevén o evitan accidentes en fábrica, donde se presenta alto tráfico de montacargas y personal, y más cuando es necesario instalar cortinas antipartículas al ingreso-salida de las bodegas que impiden la visibilidad de parte del personal o vehículos. Este problema lleva a considerar soluciones con uso de dispositivos electrónicos.

La innovación en las empresas productoras por los sistemas de semaforización electrónica produce un correcto flujo vehicular, sin afectar el dinamismo de la producción, tema poco explorado a nivel local.

Se presenta al PSoC, como una alternativa, frente a los microcontroladores más conocidos como lo son PIC y Motorola, en el diseño de dispositivos en sistemas electrónicos [10-11].

\section{Conclusiones}

- El sistema tiene como limitante que no hace discriminación entre personas o animales y los montacargas, lo cual interfiere en la lógica del sistema. 
- Las cámaras tienen la capacidad de detección en tiempo real de objetos (vehículos) en reposo y movimiento que intervengan en el bucle virtual.

- Las TrafiCam ${ }^{\circledast}$ son superiores a los Zone scanner, sensores detectores de presencia, entre otros, ya que cuentan con visualización de la zona y generan en ella diversos bucles de diferentes dimensión para la detección.

- Es necesario incluir dentro del sistema de semaforización electrónica una verificación del funcionamiento de los diferentes dispositivos que lo conforman, para garantizar un óptimo funcionamiento y brindarle una mayor confiabilidad al sistema de tráfico.

- Es importante para el éxito del funcionamiento dar sugerencias en aspectos externos al sistema electrónico, como lo son la delimitación con pintura especial para tráfico de las zonas que detecta la cámara, a fin de que los operarios no interfieran en la lógica de los semáforos, ya que este sistema está diseñado en principio para los montacargas.

- Pensar en la seguridad que brinda el sistema tanto al operario de la montacarga como al peatón intruso importa cuando hay la posibilidad de inminente choque por la cercanía del automotor-peatón, afuera o adentro de la línea de producción. Por ello se activa una sirena para evitar accidentes.

- La seguridad industrial y la delimitación de las zonas por donde deben transitar los peatones, con un propósito educativo y pedagógico, beneficia al sistema y desafecta la correcta funcionalidad del mismo.

\section{Referencias}

[1] A. Velásquez, Desarrollo de una metodología para el control de la señalización del tráfico y el sistema de semaforización utilizando las líneas de potencia eléctrica-PLT $(P L C)$, tesis de maestría, Bogotá: Universidad Nacional de Colombia, Facultad de Ingeniería, 2009. [En línea]. Disponible en: http://www.bdigital.unal.edu. co/2450/1/299913.2009.01.pdf

[2] R. Ashby, "Designer's Guide to the Cypress PSoC ${ }^{\mathrm{mm}}$," Newness, 2da edición, Burlington MA: Elsevier, 2005.

[3] O. Bailey, "Embedded systems: desktop integration", 2da. edición, Plano Texas: Wordware, Cap.10, 2005, pp. 397-421.

[4] P. Micakovic. Architecture and Programming of PSoC Microcontrollers, 1ra. edición, San Francisco, CA: New Electronic, 2000.

[5] Traficon, "Video detección para intersecciones", Dic., 2009. [En línea]. Disponible en: http://www.traficam. com/animatie.html

[6] Cypress Perform, "CY8C29x66", Ago., 2010. [En línea]. Disponible en: http://www.cypress. $\mathrm{com} /$ ? id $=1368 \& \operatorname{tab} I D=38382$

[7] Andinatraffic, Nov., 2010. [En línea]. Disponible en: http://www.andinatraffic.com/project/andina/revista/ revista.cfm

[8] Siemens Movility, "Movilidad”, Sep., 2010. [En línea]. Disponible en: http://www.mobility.siemens.com/mobility/global/en/Pages/siemens-mobility.aspx

[9] Pepperl+Fuchs, "Sensores de proximidad", Sep., 2010. [En línea]. Disponible en: http://www.pepperl-fuchs. com/global/en/classid_142.htm

[10] Multicontrol, “Sensores”, Sep., 2010. [En línea]. Disponible en: http://www.multicontrol.com.co/cms/Default.asp?Page $=33$

[11] Colombia Leds, "Semáforo vehicular", Sep., 2010. [En línea]. Disponible en: http://www.colombialeds.com/ index.php?page $=$ item $\&$ categoria $=$ Otros $\&$ item $=51420$ \&buscar $=$ 